\title{
Age-related frailty and its association with biological markers of ageing
}

\author{
Arnold Mitnitski ${ }^{1}$, Joanna Collerton², Carmen Martin-Ruiz ${ }^{3}$, Carol Jagger ${ }^{2}$, Thomas von Zglinicki ${ }^{4}$, \\ Kenneth Rockwood ${ }^{5}$ and Thomas B. L. Kirkwood ${ }^{4 *}$
}

\begin{abstract}
Background: The relationship between age-related frailty and the underlying processes that drive changes in health is currently unclear. Considered individually, most blood biomarkers show only weak relationships with frailty and ageing. Here, we examined whether a biomarker-based frailty index (FI-B) allowed examination of their collective effect in predicting mortality compared with individual biomarkers, a clinical deficits frailty index (FI-CD), and the Fried frailty phenotype.

Methods: We analyzed baseline data and up to 7-year mortality in the Newcastle $85+$ Study ( $n=845$; mean age 85.5 ). The FI-B combined 40 biomarkers of cellular ageing, inflammation, haematology, and immunosenescence. The Kaplan-Meier estimator was used to stratify participants into FI-B risk strata. Stability of the risk estimates for the FI-B was assessed using iterative, random subsampling of the $40 \mathrm{FI}-\mathrm{B}$ items. Predictive validity was tested using Cox proportional hazards analysis and discriminative ability by the area under receiver operating characteristic (ROC) curves.
\end{abstract}

Results: The mean FI-B was 0.35 (SD, 0.08), higher than the mean FI-CD $(0.22$; SD, 0.12); no participant had an FI-B score $<0.12$. Higher values of each $\mathrm{Fl}$ were associated with higher mortality risk. In a sex-adjusted model, each one percent increase in the FI-B increased the hazard ratio by $5.4 \%(\mathrm{HR}, 1.05 ; \mathrm{Cl}, 1.04-1.06)$. The Fl-B was more powerful for mortality prediction than any individual biomarker and was robust to biomarker substitution. The $\mathrm{ROC}$ analysis showed moderate discriminative ability for 7-year mortality ( $\mathrm{AUC}$ for $\mathrm{FI}-\mathrm{CD}=0.71$ and $\mathrm{AUC}$ for $\mathrm{FI}-\mathrm{B}=0.66$ ). No individual biomarker's AUC exceeded 0.61. The AUC for combined FI-CD/FI-B was 0.75 .

Conclusions: Many biological processes are implicated in ageing. The systemic effects of these processes can be elucidated using the frailty index approach, which showed here that subclinical deficits increased the risk of death. In the future, blood biomarkers may indicate the nature of the underlying causal deficits leading to age-related frailty, thereby helping to expose targets for early preventative interventions.

Keywords: Ageing, Biomarkers, Cellular ageing, Deficit accumulation, Frailty, Frailty index, Frailty phenotype, Immunosenescence, Inflammation, Newcastle 85+ study

\section{Background}

The ageing process is characterized by declining functional capacity and increasing vulnerability to disease, disability, and death. This is driven by the gradual, lifelong accumulation of molecular and cellular defects [1]. Individuals of a given chronological age vary, however, in how far these processes have advanced. Some enter a

\footnotetext{
* Correspondence: tom.kirkwood@ncl.ac.uk

${ }^{4}$ Institute for Cell and Molecular Biosciences and Newcastle University Institute for Ageing, Newcastle upon Tyne NE4 5PL, UK

Full list of author information is available at the end of the article
}

state of increased risk, compared with others of the same age, known as frailty [2]. Connecting the clinical state of age-related frailty with underlying biological age, as measured by biomarkers, has long been an elusive goal.

Frailty can be evaluated, and health status quantified, by the number of health deficits that individuals accumulate. Specifically, the frailty index (FI) is the ratio of the deficits present in a person to the total number of potential deficits evaluated [3]. The FI has been used in many epidemiological and clinical studies to grade the degree of risk of several adverse outcomes, including mortality, 
health service use, hospital-acquired complications, worsening health, and loss of independence [4-9], as well as conditions such as cognitive impairment [10], heart disease [11], osteoporosis [12], intellectual disability [13], and systemic sclerosis [14]. In most studies, the FIs have been made up of clinically-assessed health deficits [9].

In a comprehensive study of biomarkers of cellular ageing, inflammation, and immunosenescence, Collerton et al. [15] identified several biomarkers associated with frailty (assessed both by the FI and the Fried frailty phenotype [16]), where each biomarker was considered separately from the others. Although such an analysis can identify important specific effects, it is known that complex interactions between ageing mechanisms and biomarkers are likely $[17,18]$. Within the present work, we significantly extend our previous analyses by combining different biomarker measures into a FI, which may make it possible to discern a systemic effect that might be masked when the variables are used in isolation [19].

In principle, a FI of clinical health deficits should reflect the accumulation of damage at the level of the organism due to impairment of macroscopic and microscopic repair processes [20]. We were therefore interested to know whether combining biomarkers of deficits at the levels of tissue (anaemia, inflammatory markers) and cells (e.g., DNA damage, telomere length) would add to the ability of a FI based on clinical deficits (Clinical Deficits Frailty Index; FI-CD) to predict adverse outcomes.

Our objectives were 1) to develop a FI based on biomarkers (Biomarker Frailty Index; FI-B); 2) to investigate the robustness of the FI-B in relation to the specific biomarkers included; and 3) to test its predictive value in relation to death, compared to other frailty measures (FI-CD, Fried phenotype) and individual biomarkers.

\section{Methods \\ Participants}

As detailed elsewhere, the Newcastle $85+$ Study is a population-based cohort study of people born in 1921, aged 85 at recruitment, living in Newcastle upon Tyne and North Tyneside (North East England); the cohort was socio-demographically representative of the local population and of England and Wales [21-23]. The research complied with the requirements of the Declaration of Helsinki. Ethical approval was obtained from the Newcastle and North Tyneside 1 Research Ethics Committee (reference number 06/Q0905/2). Written informed consent was obtained from participants and where people lacked capacity to consent, for example because of dementia, a formal written opinion was sought from a relative or carer. Participants could decline any or all elements of the protocol.

\section{Data sources}

A multidimensional health assessment was carried out in the participant's usual residence by a research nurse. Data on pre-existing diseases and prescribed medications were obtained from general practice medical records.

\section{Blood-based biomarkers}

Biomarkers were measured from a blood sample drawn between 7 am and 10:30 am following an 8 h overnight fast and delivered to the laboratory for initial processing within 1 hour of draw. The 40 biomarkers investigated included inflammatory, haematological, immunological, cell senescence, genetic, and epigenetic markers, and were analyzed according to either previously reported methods [24] or those described in Additional file 1: Material and Methods.

\section{Recoding (dichotomizing) biomarkers as deficits}

Each biomarker was measured on a continuous scale and values were dichotomized into 'deficit' versus 'no deficit' using empirical cut points, chosen to achieve the best separation of survival curves between people with and without the deficit by minimizing the $P$ value of the log rank test (Table 1, Additional file 1: Figure S1).

\section{Frailty measures}

The FI-B was constructed by combining results for up to 40 biomarkers. For each dichotomized biomarker, a standard procedure was followed such that zero equals the absence of the deficit and 1 equals its presence. For any individual participant, the number of deficits was summed and divided by the number of potential deficits evaluated. In consequence, an individual with no deficits would have an $\mathrm{FI}=0$, and someone with every deficit present would have an $F I=1$, although previous work has shown that empirically the ceiling for FIs is generally observed at a score of 0.7 or less [4]. The FIs were calculated only if more than $80 \%$ of the component variables were available for a given individual. The FI-B was calculated in 777 participants. For illustrative purposes, we considered four FI-B strata (low, low-to-intermediate, intermediate-to-high, and highest risk of mortality) defined using empirical cut-points of $0.25,0.38$, and 0.50 , respectively, based on maximum separation of mortality curves.

The FI-CD had been calculated earlier from 40 clinical variables in 811 participants [15]. In the same previous work, the Fried frailty phenotype had also been derived in 552 participants (the chief reason why the sample for the Fried frailty phenotype was substantially lower that for the FIs was the exclusion, as per the stipulated Fried methodology, of participants with conditions which might cause them to score as frail as a result of that condition alone; in brief, reasons for exclusion were stroke, Parkinson's 
Table 1 Individual biomarkers used to compose the frailty indices ( $\mathrm{Fl}-\mathrm{B}$ ). The cut off points were defined to achieve the best separation of survival curves between people with and without the deficit and minimizing the $P$ value of the log rank test

\begin{tabular}{|c|c|c|c|c|c|}
\hline \multirow[t]{2}{*}{ Biomarker } & \multirow{2}{*}{$\begin{array}{l}\text { Cut off } \\
\text { point }\end{array}$} & \multirow{2}{*}{$\begin{array}{l}\text { Direction } \\
\text { of risk }\end{array}$} & \multicolumn{2}{|c|}{ Number of participants } & \multirow[t]{2}{*}{$P$ value** } \\
\hline & & & At lower risk & At higher risk & \\
\hline \multicolumn{6}{|l|}{ Inflammation* } \\
\hline Cytomegalovirus serology (lgG) & Positive & & 108 & 641 & 0.327 \\
\hline High sensitivity C-reactive protein (mg/L) & $>25$ & High & 737 & 37 & $<0.001$ \\
\hline IL-6, basal (pg/mL) & $>15$ & High & 27 & 676 & 0.399 \\
\hline IL-6, post-stimulation (pg/mL) & $>1100$ & High & 176 & 527 & 0.92 \\
\hline TNF-alpha, basal (pg/mL) & $<95$ & Low & 55 & 637 & 0.646 \\
\hline TNF-alpha, post-stimulation (pg/mL) & $>80$ & High & 60 & 643 & 0.116 \\
\hline Leptin (ng/mL) & $<40$ & Low & 214 & 495 & 0.023 \\
\hline Adiponectin $(\mu \mathrm{g} / \mathrm{mL})$ & $>20$ & High & 657 & 83 & 0.012 \\
\hline Homocysteine $(\mu \mathrm{mol} / \mathrm{L})$ & $>30$ & High & 422 & 35 & 0.006 \\
\hline Albumin (g/L) & $<40$ & Low & 365 & 397 & $<0.001$ \\
\hline \multicolumn{6}{|l|}{ Haematological } \\
\hline Haemoglobin (g/dL) & $<11$ & Low & 676 & 76 & $<0.001$ \\
\hline Platelets $\left(\times 10^{9} / \mathrm{L}\right)$ & $<170$ & Low & 675 & 73 & 0.003 \\
\hline White blood cells $\left(\times 10^{9} / L\right)$ & $>7$ & High & 470 & 274 & 0.035 \\
\hline Neutrophils $\left(\times 10^{9} / \mathrm{L}\right)$ & $>7$ & High & 715 & 29 & 0.007 \\
\hline Lymphocytes $\left(\times 10^{9} / \mathrm{L}\right)$ & $<1.5$ & Low & 530 & 214 & 0.028 \\
\hline Monocytes $\left(\times 10^{9} / \mathrm{L}\right)$ & $>0.75$ & High & 639 & 105 & 0.14 \\
\hline Basophils $\left(\times 10^{9} / \mathrm{L}\right)$ & $<0.06$ & Low & 156 & 588 & 0.156 \\
\hline Eosinophils $\left(\times 10^{9} / \mathrm{L}\right)$ & $>0.5$ & High & 688 & 56 & 0.15 \\
\hline \multicolumn{6}{|l|}{ Immunosenescence } \\
\hline CD4 T cells (\% T cells) & $<44$ & Low & 345 & 367 & 0.029 \\
\hline CD8 T cells (\% T cells) & $>35$ & High & 579 & 128 & 0.015 \\
\hline CD8 TEMRA T cells (\% CD8 T cells) & $>0.6$ & High & 672 & 36 & 0.014 \\
\hline Senescent Memory CD4 T cells (\% Memory CD4 T cells) & $>70$ & High & 618 & 93 & 0.079 \\
\hline Memory CD4 T cells (\% CD4 T cells) & $>35$ & High & 672 & 43 & 0.057 \\
\hline CD4/CD8 T cell ratio & $<0.6$ & Low & 662 & 31 & 0.005 \\
\hline Memory/naïve CD4 T cell ratio & $>3.3$ & High & 692 & 20 & 0.034 \\
\hline Memory/naïve CD8 T cell ratio & $>15$ & High & 677 & 20 & 0.352 \\
\hline Memory/naïve B cell ratio & $>2.5$ & High & 652 & 36 & 0.026 \\
\hline \multicolumn{6}{|l|}{ Cellular ageing/Oxidative stress } \\
\hline Telomere length (bp) & $<3000$ & Low & 634 & 66 & 0.118 \\
\hline DNA repair (\%) & $<20$ & Low & 589 & 156 & 0.078 \\
\hline DNA damage/DNA repair ratio & $>6.5$ & High & 688 & 44 & 0.063 \\
\hline TGF beta, transforming growth factor beta $(\mathrm{ng} / \mathrm{mL})$ & $<20$ & Low & 102 & 644 & 0.003 \\
\hline IGFBP1, insulin-like growth factor-binding protein 1 ( $\mathrm{ng} / \mathrm{mL})$ & $>150$ & High & 574 & 162 & 0.001 \\
\hline IGFBP3, insulin-like growth factor-binding protein $3(\mathrm{ng} / \mathrm{mL})$ & $<800$ & Low & 92 & 652 & 0.357 \\
\hline iPF2alpha-III (LC/MS/MS) (ng/mL) & $<4.5$ & Low & 85 & 629 & 0.053 \\
\hline iPF2alpha-VI (LC/MS/MS) (ng/mL) & $<4$ & Low & 423 & 291 & 0.1 \\
\hline iPF2alpha-III (AutoDELFIA) (ng/mL) & $<0.6$ & Low & 512 & 103 & 0.258 \\
\hline \multicolumn{6}{|l|}{ Genetic/Epigenetic } \\
\hline Mitochondrial DNA haplogroup & $\mathrm{X}, \mathrm{l}$, hete & lasmic & 675 & 30 & 0.031 \\
\hline
\end{tabular}


Table 1 Individual biomarkers used to compose the frailty indices ( $\mathrm{Fl}-\mathrm{B}$ ). The cut off points were defined to achieve the best separation of survival curves between people with and without the deficit and minimizing the $P$ value of the log rank test (Continued)

\begin{tabular}{|c|c|c|c|c|c|}
\hline APOE genotype & E4 & & 454 & 167 & 0.024 \\
\hline CPG island DNA methylation (\%) & $>7$ & High & 28 & 451 & 0.67 \\
\hline Line1 DNA methylation, surrogate for genome-wide DNA methylation (\%) & $>80$ & High & 73 & 197 & 0.63 \\
\hline
\end{tabular}

*The recorded cytokine levels may be perceived as higher than usual. Because we wanted to measure basal and post-stimulated cytokines, we measured cytokines in non-coagulated LiHe blood, using blood supernatants, not serum. Furthermore, we used an electrochemiluminescent method (MSD technology) that in our hands is much more sensitive than standard ELISA assays. Both factors are likely to explain our observed cytokine levels

**Statistically significant P-values $(\leq 0.05)$ are shown in bold

disease, mini-mental state examination score of less than 18, or taking drugs for dementia, Parkinson's disease, or depression).

\section{Data analysis}

Kaplan-Meier survival and Cox proportional hazard models were used to estimate the probability of survival, in which FI values were converted to $0-100$ integers by rounding them after multiplying them by 100 , giving equal percent increments for modelling. To evaluate the robustness of the separation of the FI-B strata, we randomly selected up to 30 out of 40 biomarkers/deficits and repeated the Kaplan-Meier survival analysis 1,000 times. We also compared different versions of the FI-B to address whether effects were cumulative or driven by just a few biomarkers.

The hazard ratios (HR) of the FIs (FI-B and FI-CD) were adjusted for sex, and considered separately and together. Receiver operating characteristic (ROC) analysis was used to assess the discriminative ability of the FIs, separately and in combination, as well as the Fried frailty phenotype and individual biomarkers, in relation to mortality. The confidence intervals for the ROC were calculated using bootstrapping, with 1,000 replications. Data analysis was conducted using SPSS Version 21 (IBM SPSS.) The statistical significance level was set at $P<0.05$.

\section{Results}

\section{Biomarker-based frailty index (FI-B)}

The mean age of the sample of 777 people in whom the FI-B could be calculated was 85.5 years (SD, 0.4). Most were women (60.9\%). The FI-B and FI-CD samples did not differ significantly in sex, years of education, percent smokers, body mass index, or cognition (Additional file 1: Table S2).

The FI-B showed a slightly skewed distribution, fitted by the gamma density function with shape and scale parameters of 18.77 and 0.02, respectively (Fig. 1, Panel b). The 5th centile of the FI-B was 0.24, and the 95th and 99th centiles were 0.48 and 0.60 , respectively. The histogram of the FI-CD was more skewed with a longer right tail (Fig. 1, Panel a); the gamma fit shape and scale parameters
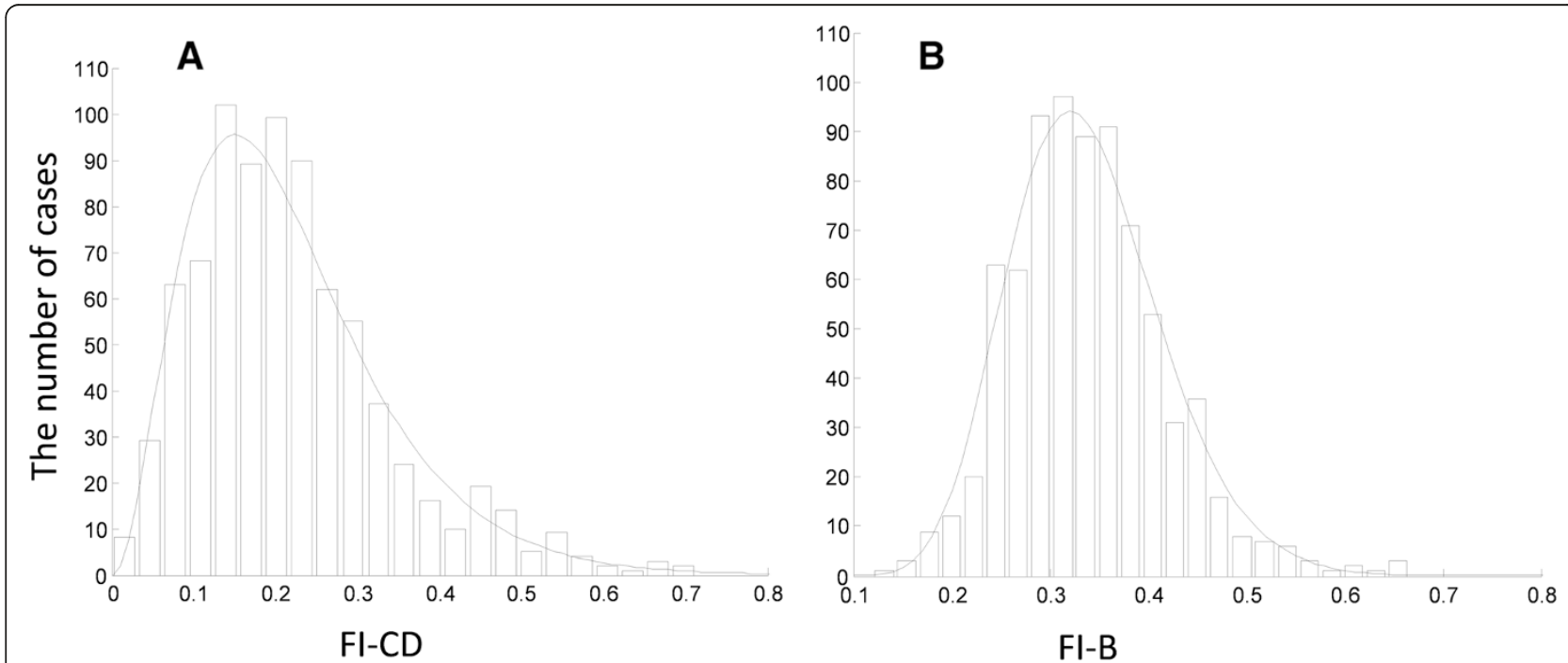

Fig. 1 Histograms of the a Clinical Deficit Frailty Index (FI-CD) and $\mathbf{b}$ Biomarker Frailty Index (FI-B), and the best fit gamma density functions (solid lines) with the parameters of shape and scale 18.77 and 0.02 for FI-CD and 3.24 and 0.07 for FI-B, respectively 
were 3.24 and 0.07 , respectively. The 5 th centile of the FI-CD was 0.06 , and the 95th and 99th centiles were 0.46 and 0.59 , respectively. The mean FI-B was 0.35 (SD, 0.08), higher than the mean FI-CD of 0.22 (SD, 0.12). Individual values of the FI-B were weakly correlated with the FI-CD $(\mathrm{r}=0.16, P<0.001)$. On average, the clinically fittest people (those with 0 or 1 clinical deficits, i.e., FI-CD $=0$ or 0.02 ) had an average FI-B value of 0.33 (CI, 0.32-0.34), compared with an average FI-B value of 0.39 (CI, 0.36-0.41) for the clinically frailest people (FI-CD $>0.5$ ).

Of the 40 biomarkers used to compose the FI-B, 21 showed significant differences $(P<0.05)$ in mortality between people with and without each deficit (Table 1). Of those 21 biomarkers, nine demonstrated a high separation of survival curves, with $\log \operatorname{rank} P<0.001$.

The FI-B was strongly associated with 7-year mortality. In the Cox proportional hazards model adjusted for sex (female sex is protective; HR, 0.73; CI, 0.60-0.88), each one percent increase in FI-B was associated with $5.4 \%$ increase in the hazards ratio (HR, 1.05; CI, 1.04-1.07). Likewise, the Kaplan-Meier survival curves showed the effect of increasing frailty across the four FI-B strata (Fig. 2a). This pattern was robust: random sub-samplings of 30 biomarkers out of the 40 available also showed good separation between the four strata, with only little overlap between neighbouring groups (Fig. 2b). With decreasing numbers of biomarkers included, the overlap between the groups greatly increased (Additional file 1: Figure S2). Notably, amongst those who clinically were not frail (FI-CD scores in the lowest quartile) having an FI-B higher than median (0.33) was associated with much higher mortality (Fig. 3).

\section{Comparing different versions of the FI-B}

We investigated three additional versions of the FI-B. The first (FI-B-9) was calculated from the nine biomarkers that were individually highly significantly $(P<0.001)$ associated with mortality in univariate analysis. Likewise, the FI-B-21 was calculated from all 21 significant $(P<0.05)$ biomarkers and the third group (FI-B-NS-19) from the remaining 19 biomarkers that individually had shown no significant association with mortality $(P>0.05)$. In the Cox regression analysis, each FI-B (comprised from 9, 21, and 19 items) was significantly associated with mortality. The strongest association was seen with the FI-B-9 (HR, 1.03; CI, 1.03-1.04) and the FI-B-21 (HR, 1.03; CI, 1.03-1.04) followed by the FI-B-NS-19 (HR, 1.01; CI, 1.01-1.02). These relationships did not change after adjustment for sex and the FI-CD.

\section{Performance of the various frailty measures}

The ROC analysis showed moderate discriminative ability in relation to 7-year mortality for the different frailty measures (Table 2). The lowest area under the curve (AUC) value for the FI-B versions was for the FI-B-NS19 (AUC = 0.60). The FI-CD had the highest predictive accuracy, with $\mathrm{AUC}=0.71$. The AUC further increased when both the FI-B and FI-CD were entered into the model (AUC $=0.75)$, and slightly but insignificantly increased after adjusting for sex $(\mathrm{AUC}=0.77)$. Interestingly,

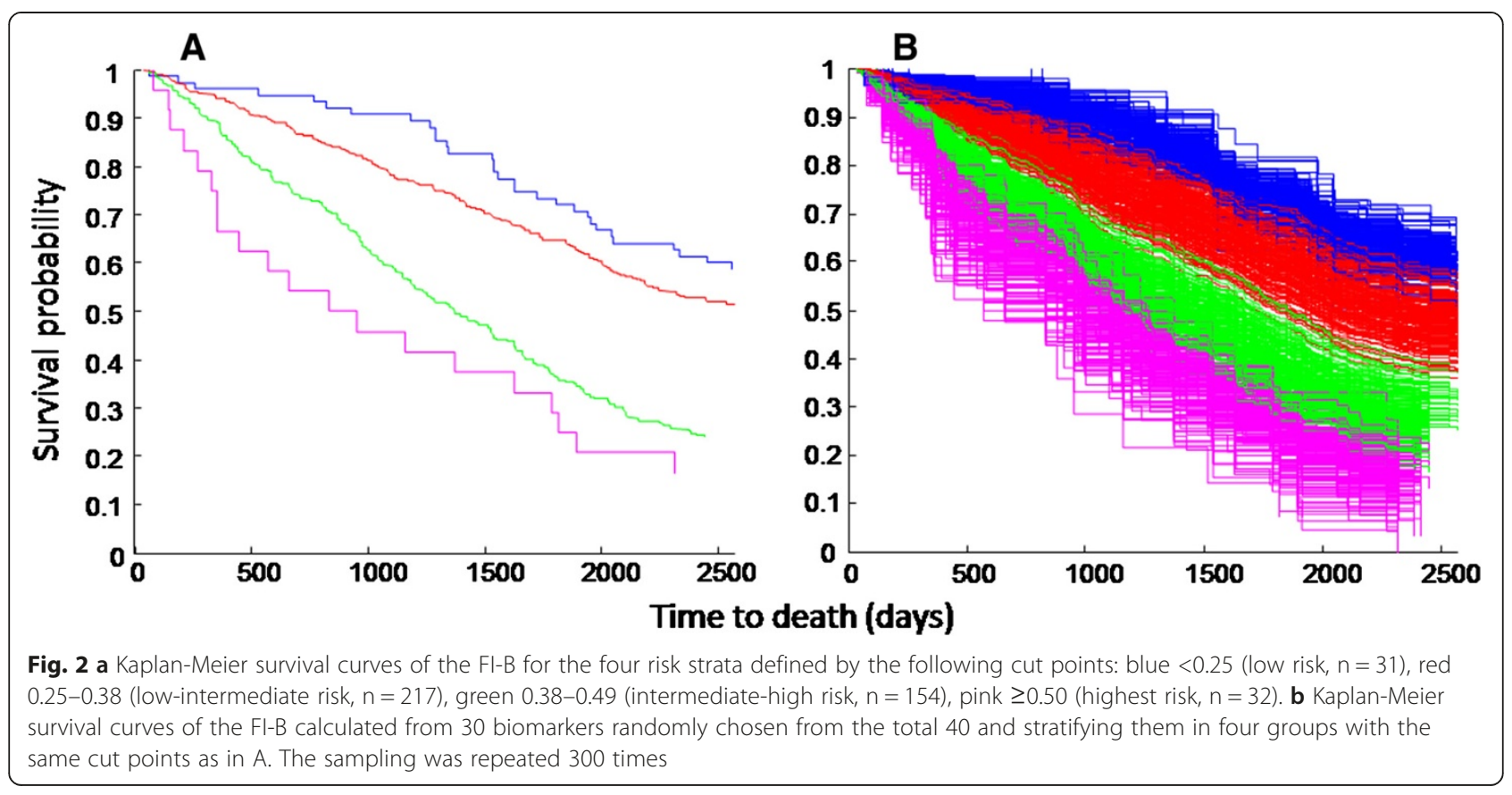




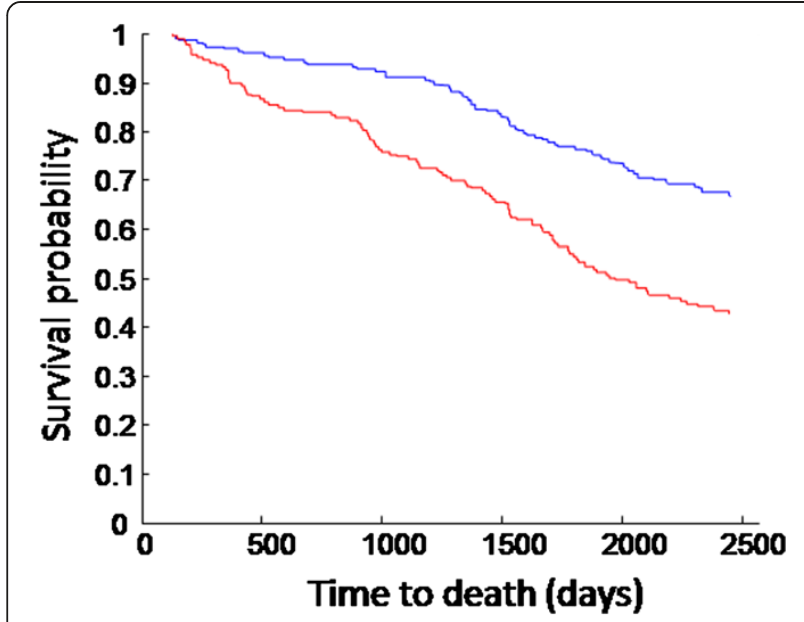

Fig. 3 Survival of people who were not clinically frail (FI-CD $<$ lower quartile) differs by the value of their FI-B: red for those with higher than median Fl-B and blue for those with lower than median FI-B

the biomarkers considered individually had rather poor discriminative ability: the AUC did not exceed 0.61 (CI, 0.52-0.64; albumin).

Not included in Table 2 is the AUC value obtained for the Fried frailty phenotype, which was 0.58 (95\% CI, 0.53-0.63), slightly (but not significantly) lower than the lowest AUC value for the FI-B versions (FI-B-NS-19; $\mathrm{AUC}=0.60)$. Since the exclusions that were necessary to produce the data for the Fried frailty phenotype might have resulted in a generally healthier subsample, which could potentially have biased the comparison, the ROC analyses for the various frailty indices were also performed using only this subsample (Additional file 1: Table S3). When this was done the predictive accuracy of the various FIs was reduced but they retained higher AUC values than for the Fried frailty phenotype. Adding the Fried phenotype to FI-CD and FI-B did not change the AUC.

\section{Discussion}

\section{Overview of the findings}

In this population-based sample of very old people, combining even 'small-effect' biomarkers in a FI resulted

Table 2 The area under the receiving operating characteristic (AUC) with $95 \%$ confidence intervals for different versions of the frailty index $(\mathrm{Fl})$

\begin{tabular}{lll}
\hline & AUC & $95 \% \mathrm{Cl}$ \\
\hline FI-B & 0.66 & $0.62-0.69$ \\
FI-B-21 & 0.68 & $0.62-0.73$ \\
FI-B-9 & 0.65 & $0.61-0.69$ \\
FI-B-NS & 0.60 & $0.52-0.64$ \\
FI-CD & 0.71 & $0.67-0.74$ \\
FI-CD + Fl-B & 0.75 & $0.71-0.78$ \\
FI-CD +FI-B + sex & 0.77 & $0.74-0.81$ \\
\hline
\end{tabular}

in a strong association with mortality. A FI produced from 40 biomarkers (and variants of it, based on statistical criteria) stratified people into four mortality risk groups. The FI-B was robust to inclusion/exclusion of individual biomarkers: any 30 randomly selected biomarkers demonstrated the same patterns of relationship to death. Such patterns became degraded, with greater overlap between the neighbouring groups, with decreasing numbers of biomarkers included (Additional file 1: Figure S2). The combination of the FI-B and a clinical FI, FI-CD, significantly improved mortality prediction compared to either index alone.

\section{Comparing the $\mathrm{FI}-\mathrm{B}$ and $\mathrm{FI}-\mathrm{CD}$}

Although the FI-B and FI-CD were significantly but weakly correlated, they showed different distributions. On average, the clinically fittest people (those with 0 or 1 clinical deficits, i.e., FI-CD, 0 or 0.02 ) had an average FI-B value of 0.33 (CI, 0.32-0.34). The 95th and 99th centiles for both frailty indices were very similar $(0.46$ for FI-CD and 0.48 for FI-B for the former; 0.59 for FI-CD and 0.60 for the latter), whereas the 5 th centiles were different (0.06 for FI-CD vs. 0.24 for FI-B). This suggests that, at low FI-CD values, the FI-B is a more sensitive measure of health, i.e., it indicates important subclinical deficit accumulation (Fig. 3) whereas the frailest group is frail by either measure. The observation that FI-B is a stronger predictor of mortality in the group who were not clinically frail points to a question: do people with low FI-CD and high FI-B become clinically frail before death? To answer this will require follow-up data on the people who were non-frail in an earlier phase of assessment. Even so, the FI-B was not as closely associated with mortality as was the FI-CD, suggesting that the added value of biomarkers is highest in people without detectable clinical deficits. Note too that in both distributions, the right tail shows that $99.5 \%$ of people had an FI value $<0.7$ in either the FI-B or the FI-CD. The reproducibility of this observation [7, 25-28] is consistent with a system property that has yet to be elucidated.

\section{Combining the Fls}

Combining the FI-B and FI-CD resulted in an increase in their discriminative ability $(\mathrm{AUC}=0.76$; compared to 0.68 for FI-B and 0.71 for FI-CD, separately). This indicates that the indices are complementary to each other. At this age, the clinically fittest people as measured by the FI-CD, still have an important number of biomarker abnormalities, as reflected by their high mean FI-B scores of 0.32 , well above the usual levels in a FI-CD of $0.20-0.25$ that would define a person as "frail" using a dichotomous definition. Even so, changes that are subclinical alone appear to be less related to mortality; to what extent they predict adverse outcomes other than mortality will be of 
interest. Interestingly, the AUC for the combination of FI-CD with the FI-B-NS-19 and adjusted for sex was 0.75 , suggesting further that the additive effect of items is important, even for those that individually might not be statistically significant.

\section{IL-6 and TNFa data}

It was interesting that neither basal nor stimulated noncoagulated cytokine production were associated with 7 -year survival. This is in accordance with our earlier report of absence of an association between these inflammatory markers and 18-month survival [24], although basal IL-6 was earlier associated with frailty (both Fried frailty and the FI-CD) [15]. We are aware that serum IL-6 and TNFo levels have been associated with survival in other studies. There are a number of speculations possible regarding the comparative predictive power of cell-based versus serumbased measures, but we believe these would be premature at this stage.

\section{Strengths and limitations}

Our data must be interpreted with caution. Often, abnormalities are expressed only in one direction (i.e., only values above (or below) a reference level are considered 'abnormal') [15]. Our approach was strictly empirical: for each dichotomized biomarker variable, we tested whether the highest or lowest scores were associated with mortality. The potential for increased risk at both ends of the normal range has been observed for several characteristics [29-31]. Even so, we did not find evidence of a U-shaped risk of mortality for any of these biomarkers (Table 1). Here, whichever direction was associated with increased mortality was scored as a deficit for that item (Table 1 and Additional file 1: Figure S1), as has been done for all versions of the FI. This method of identifying cut-points is likely to be related to mortality within studies, so that it is not clear how generalizable it is to other populations. This needs to be evaluated. On the other hand, percentilebased cut-points, e.g., tertiles [32] or twentieth percentile rule [33], also depend on the population sample considered in those studies. Often, such cut-points are not well established, so that we were interested to note that our algorithm-identified cut-point for telomere length was virtually identical to what others have suggested [34-36]. The fact that we used mortality data to establish cutpoints in order to dichotomise the biomarker values, and later used the resulting aggregate FIs to predict mortality, could also be considered a limitation. However, this was necessary given the lack of independent data, and any distortion is likely to have been small. As future data accrue, it should become possible for cut-point determination to be done on one population and prediction on another. Participants were all over the age of 85 years, which can limit generalizability. On the other hand, there is a high prevalence of clinical frailty in this under-investigated group. Furthermore, at this age, for a modest sample size and budget - many events are likely to accrue.

Our study has several strengths. An important aspect of this study is the population-based nature of the sample [15]. In addition to the sample size and the range of biomarkers considered, it is the first study in which the FI approach has been applied to biomarkers. The FI-B shared many properties with the FI-CD, although it adds important value to the latter in identifying people at increased risk of death. The availability of a large number of biomarkers in this study allowed us to make a direct comparison of the different frailty measures, and also to assess the robustness of the FI-B to its composition of individual biomarkers. We have indicated how the algorithm for biomarker cut-points can be defined, which, coupled with earlier publications $[9,37,38]$ on a standard procedure for creating a FI, allows for replication of the results by others.

\section{Frailty and ageing: conceptualisation}

Although, as generally agreed, frailty is an age-related state of vulnerability to stressors, there are different ways to operationalize it. Frailty as defined by a clinical phenotype exposes the consequences of functional deterioration and indicates proximate factors that render the frail individual more likely to die. By contrast, if frailty can be related to biological markers of aging, these relationships should come closer to identifying the underlying factors that give rise to these functional declines. Within the frailty phenotype approach, the focus has been on clinical features of frailty; while it has been acknowledged that these need ultimately to be understood in terms of biological mechanisms, the connection has not yet been established. On the other hand, the FI approach has been chiefly one based on pragmatic scoring of multiple clinical deficits, to which subclinical ones can be added [39]. Thus, its potential utility is that the FI-B could be used as a pre-clinical measure to identify at-risk individuals before changes are apparent on clinical examination. The FI-B might also help to inform the mechanistic connections between frailty and the underlying biology of intrinsic aging. This framework also allows a practical means of addressing a fundamental fact of old age, which is, as pointed out in a recent Nature commentary [40], that its problems come as a package.

\section{Conclusions}

Combining biomarkers in a FI, even those not individually associated with mortality, improves mortality prediction much beyond that of individual biomarkers. Additionally, combining a FI based on biomarkers with one based on clinically detectable deficits further improves the performance in identifying vulnerable people who are at risk of 
death. Finally, that so many items can contribute to mortality prediction reflects the systemic nature of ageing and mortality - health status depends on the systemic effect of multiple characteristics, of which those located within the individual can be summarized in a FI.

\section{Additional file}

Additional file 1: Figure S1. Kaplan-Meier survival curves of the individual biomarkers split by two groups using the cut points chosen to achieve the best separation of survival curves between people with and without the deficit and minimizing the p-value of the log rank test (Table 1). Red lines show survival curves for the group "at higher risk" and the blue lines are for "at lower risk" individuals.

\section{Abbreviations}

AUC: Area under the curve; FI: Frailty index; FI-B: Frailty index based on biomarkers; FI-CD: Frailty index based on clinical deficits; HR: Hazard ratios; ROC: Receiver Operating Characteristic.

\section{Competing interests}

The authors declare that they have no competing interests.

\section{Authors' contributions}

AM, JC, and CJ carried out the analyses of frailty indices. CMR and TVZ performed and planned the biomarker assays. JC, TBLK, AM, and KR participated in the design of the study. TBLK conceived of the Newcastle 85+ Study and supervised its conduct. All authors contributed to, read, and approved the final manuscript.

\section{Acknowledgements}

The work was supported by the UK Medical Research Council and the Biotechnology and Biological Sciences Research Council (G0500997; G0601333) (JC, CMR, CJ, TBK, TVZ); the NIHR Biomedical Research Centre based at Newcastle upon Tyne Hospitals NHS Foundation Trust and Newcastle University (CMR); and the Dunhill Medical Trust and an operating grant from the Canadian Institutes of Health Research (MOP25388) (AM, KR). The funders had no role in the study design, collection, analysis and interpretation of data, in writing the paper, or in the decision to submit the paper for publication.

\section{Author details}

${ }^{1}$ Department of Medicine, Dalhousie University, Halifax, NS B3H 2E1, Canada. ${ }^{2}$ Institute of Health and Society and Newcastle University Institute for Ageing, Newcastle upon Tyne NE4 5PL, UK. Institute of Neuroscience and Newcastle University Institute for Ageing, Newcastle upon Tyne NE4 5PL, UK. ${ }^{4}$ Institute for Cell and Molecular Biosciences and Newcastle University Institute for Ageing, Newcastle upon Tyne NE4 5PL, UK. ${ }^{5}$ Division of Geriatric Medicine, Dalhousie University, Halifax, NS B3H 2E1, Canada.

Received: 23 February 2015 Accepted: 12 June 2015

Published online: 13 July 2015

\section{References}

1. Kirkwood TB. Understanding the odd science of aging. Cell. 2005;120:437-47.

2. Clegg A, Young J, lliffe S, Rikkert MO, Rockwood K. Frailty in elderly people. Lancet. 2013;381:752-62.

3. Mitnitski AB, Mogilner AJ, Rockwood K. Accumulation of deficits as a proxy measure of aging. ScientificWorldJournal. 2001;1:323-36.

4. Rockwood K, Mitnitski A. Frailty defined by deficit accumulation and geriatric medicine defined by frailty. Clin Geriatr Med. 2011;27:17-26.

5. Goggins WB, Woo J, Sham A, Ho SC. Frailty index as a measure of biological age in a Chinese population. J Gerontol A Biol Sci Med Sci. 2005;60:1046-51.

6. Gu D, Dupre ME, Sautter J, Zhu H, Liu Y, Yi Z. Frailty and mortality among Chinese at advanced ages. J Gerontol B Psychol Sci Soc Sci. 2009;64:279-89.

7. Yang $Y$, Lee LC. Dynamics and heterogeneity in the process of human frailty and aging: evidence from the U.S. older adult population. J Gerontol B Psychol Sci Soc Sci. 2010;65B:246-55.
8. Kulminski AM, Ukraintseva SV, Culminskaya IV, Arbeev KG, Land KC, Akushevich $L$, et al. Cumulative deficits and physiological indices as predictors of mortality and long life. J Gerontol A Biol Sci Med Sci. 2008;63:1053-9.

9. Krishnan M, Beck S, Havelock W, Eeles E, Hubbard RE, Johansen A. Predicting outcome after hip fracture: using a frailty index to integrate comprehensive geriatric assessment results. Age Ageing. 2014;43:122-6.

10. Song X, Mitnitski A, Rockwood K. Nontraditional risk factors combine to predict Alzheimer disease and dementia. Neurology. 2011;77:227-34.

11. Wallace LM, Theou O, Kirkland SA, Rockwood MR, Davidson KW, Shimbo D, et al. Accumulation of non-traditional risk factors for coronary heart disease is associated with incident coronary heart disease hospitalization and death. PLoS One. 2014;9:e90475.

12. Kennedy CC, loannidis G, Rockwood K, Thabane L, Adachi JD, Kirkland S, et al. A Frailty Index predicts 10-year fracture risk in adults age 25 years and older: results from the Canadian Multicentre Osteoporosis Study (CaMos). Osteoporosis Int. 2014;25:2825-32.

13. Schoufour JD, Mitnitski A, Rockwood K, Evenhuis HM, Echteld MA. Development of a frailty index for older people with intellectual disabilities: results from the HA-ID study. Res Dev Disabil. 2013;34:1541-55.

14. Rockwood MR. MacDonald E, Sutton E, Rockwood K, Baron M; Canadian Scleroderma Research Group. Frailty index to measure health status in people with systemic sclerosis. J Rheumatol. 2014;41:698-705.

15. Collerton J, Martin-Ruiz C, Davies K, Hilkens CM, Isaacs J, Kolenda C, et al. Frailty and the role of inflammation, immunosenescence and cellular ageing in the very old: cross-sectional findings from the Newcastle $85+$ Study. Mech Ageing Dev. 2012;133:456-66.

16. Fried LP, Tangen CM, Walston J, Newman AB, Hirsch C, Gottdiener J, et al. Cardiovascular Health Study Collaborative Research Group. Cardiovascular Health Study Collaborative Research Group. Frailty in older adults: evidence for a phenotype. J Gerontol A Biol Sci Med Sci. 2001;56:M146-56.

17. Kirkwood TBL. A systematic look at an old problem. Nature. 2008;451:644-7.

18. Kirkwood TBL. Systems biology of ageing and longevity. Philos Trans R Soc Lond B Biol Sci. 2011;366:64-70.

19. Howlett S, Rockwood M, Mitnitski A, Rockwood K. Standard laboratory tests to identify older adults at increased risk of death. BMC Med. 2014;12:171.

20. Howlett SE, Rockwood K. New horizons in frailty: ageing and the deficit-scaling problem. Age Ageing. 2013:42:416-23.

21. Collerton J, Barrass K, Bond J, Eccles M, Jagger $C_{\text {, James }} \mathrm{O}$, et al. The Newcastle 85+ study: biological, clinical and psychosocial factors associated with healthy ageing: study protocol. BMC Geriatr. 2007;7:14.

22. Collerton J, Davies K, Jagger C, Kingston A, Bond J, Eccles MP, et al. Health and disease in 85 year olds: baseline findings from the Newcastle $85+$ cohort study. BMJ. 2009;339:b4904.

23. Davies K, Collerton JC, Jagger C, Bond J, Barker SA, Edwards J, et al. Engaging the oldest old in research: lessons from the Newcastle 85+ study. BMC Geriatr. 2010;10:64.

24. Martin-Ruiz C, Jagger C, Kingston A, Collerton J, Catt M, Davies K, et al. Assessment of a large panel of candidate biomarkers of ageing in the Newcastle 85+ study. Mech Ageing Dev. 2011;132:496-502.

25. Saum KU, Dieffenbach AK, Müller H, Holleczek B, Hauer K, Brenner H. Frailty prevalence and 10-year survival in community-dwelling older adults: results from the ESTHER cohort study. Eur J Epidemiol. 2014;29:171-9.

26. Rockwood K, Mitnitski A. Limits to deficit accumulation in elderly people. Mech Ageing Dev. 2006;127:494-6.

27. Bennett S, Song X, Mitnitski A, Rockwood K. A limit to frailty in very old, community-dwelling people: a secondary analysis of the Chinese longitudinal health and longevity study. Age Ageing. 2013;42:372-7.

28. Armstrong JJ, Mitnitski A, Launer L, White LR, Rockwood K. Frailty in the Honolulu-Asia aging study: deficit accumulation in a male cohort followed to $90 \%$ mortality. J Gerontol A Biol Sci Med Sci. 2015;70:125-31.

29. Abdelhafiz AH, Loo BE, Hensey N, Bailey C, Sinclair A. The U-shaped relationship of traditional cardiovascular risk factors and adverse outcomes in later life. Aging Dis. 2012;3:454-64.

30. Hubbard RE, O'Mahony MS, Savva GM, Calver BL, Woodhouse KW. Inflammation and frailty measures in older people. J Cell Mol Med. 2009;13:3103-9.

31. Okumiya K, Matsubayashi K, Wada T, Fujisawa M, Osaki Y, Doi Y, et al. A U-shaped association between home systolic blood pressure and four-year mortality in community-dwelling older men. J Am Geriatr Soc. 1999;47:1415-21.

32. Varadhan $R$, Yao W, Matteini A, Beamer BA, Xue QL, Yang H, et al. Simple biologically informed inflammatory index of two serum cytokines predicts 
10 year all-cause mortality in older adults. J Gerontol A Biol Sci Med Sci. 2014;69:165-73.

33. Romero-Ortuno R, Walsh CD, Lawlor BA, Kenny RA. A frailty instrument for primary care: findings from the Survey of Health, Ageing and Retirement in Europe (SHARE). BMC Geriatr. 2010;10:57.

34. Collerton J, Martin-Ruiz C, Kenny A, Barrass K, von Zglinicki T, Kirkwood T, et al. Newcastle $85+$ Core Study Team. Telomere length is associated with left ventricular function in the oldest old: the Newcastle 85+ study. Eur Heart J. 2007;28:172-6.

35. Woo J, Tang NL, Suen E, Leung JC, Leung PC. Telomeres and frailty. Mech Ageing Dev. 2008;29:642-8.

36. Saum KU, Dieffenbach AK, Müezzinler A, Müller H, Holleczek B, Stegmaier C, et al. Frailty and telomere length: cross-sectional analysis in 3537 older adults from the ESTHER cohort. Exp Gerontol. 2014;58:250-5.

37. Searle SD, Mitnitski A, Gahbauer EA, Gill TM, Rockwood K. A standard procedure for creating a frailty index. BMC Geriatr. 2008;8:24.

38. Parks RJ, Fares E, Macdonald JK, Ernst MC, Sinal CJ, Rockwood K, et al. A procedure for creating a frailty index based on deficit accumulation in aging mice. J Gerontol A Biol Sci Med Sci. 2012;67:217-27.

39. Whitehead JC, Hildebrand BA, Sun M, Rockwood MR, Rose RA, Rockwood K, et al. A clinical frailty index in aging mice: comparisons with frailty index data in humans. J Gerontol A Biol Sci Med Sci. 2014;69:621-32.

40. Fontana L, Kennedy BK, Longo VD, Seals D, Melov S. Medical research: treat ageing. Nature. 2014;511:405-7.

\section{Submit your next manuscript to BioMed Central and take full advantage of:}

- Convenient online submission

- Thorough peer review

- No space constraints or color figure charges

- Immediate publication on acceptance

- Inclusion in PubMed, CAS, Scopus and Google Scholar

- Research which is freely available for redistribution 